\title{
Inhibition of miR-383 suppresses oxidative stress and improves endothelial function by increasing sirtuin 1
}

\author{
Baoxiang Hu(iD, Zushun Gong (iD, and Zhaohui Bi id \\ Cardiac Intensive Care Unit, Zibo Central Hospital, Zibo, Shandong, China
}

\begin{abstract}
Previous research has shown that suppression of miR-383 can prevent inflammation of the endothelium, as well as postpone the development of atherosclerosis. However, the role of miR-383 in endothelial cell apoptosis in diabetes remains unclear. The aim of this study was to investigate the function of miR-383 in high glucose-induced apoptosis and oxidative stress in endothelial cells. A series of experiments involving qualitative polymerase chain reaction, cell transfection, luciferase assay, assessment of cell death, detection of catalase and superoxide dismutase concentrations, detection of intracellular reactive oxygen species (ROS), and western blot analysis were performed in this study. We found that miR-383 expression was promoted, while $\mathrm{NAD}^{+}$-dependent deacetylase and sirtuin 1 (SIRT1) expressions were suppressed in the endothelium of the aorta in $\mathrm{db} / \mathrm{db}$ mice as well as in human umbilical vein endothelial cells, which were treated with high glucose (HG). Increased expression of miR-383 decreased expression of SIRT1, while suppression of miR-383 promoted expression of SIRT1 in human umbilical vein endothelial cells (HUVECs). Furthermore, suppression of miR-383 following transfection with miR-383 suppressor repressed cell death and generation of ROS in HUVECs. SIRT1 knockdown by siRNA-SIRT1 reversed the suppressive effect of miR-383 inhibition on ROS production and cell apoptosis induced by HG treatment. Overall, the findings of our research suggested that suppression of miR-383 repressed oxidative stress and reinforced the activity of endothelial cells by upregulation of SIRT1 in db/db mice, and targeting miR-383 might be promising for effective treatment of diabetes.
\end{abstract}

Key words: miR-383; Oxidative stress; Human umbilical vein endothelial cells; Sirtuin 1

\section{Introduction}

Diabetes elevates the risk of cardiovascular diseases (CVD), including hypertension and atherosclerosis, due to endothelial dysfunction related to oxidative stress (OS) (1-3). In the diabetic state, excessive generation of reactive oxygen species (ROS) in the walls of vessels suppresses the bioavailability of nitric oxide (NO), decreasing endothelium-dependent relaxation and leading to severe cell injury and death $(4,5)$. Consequently, methods to attenuate OS serve as potential ways to increase the activity of endothelial cells (ECs). Sirtuin 1 (SIRT1), an $\mathrm{NAD}+$ (nicotinamide adenine dinucleotide)-dependent deacetylase, protects against oxidative stress and cell death $(6,7)$. Emerging evidence indicates that microRNAs (miRNAs) modulate SIRT1 expression in various physiologic processes including stress resistance, apoptosis, and energy balance $(8,9)$.

miRNAs, small endogenous non-coding RNAs, have been found to be biomarkers for various diseases (10). The function of miRNAs is modulated by binding to the $3^{\prime}-$ UTR of target mRNAs, resulting in degeneration of mRNA and prohibition of translation (11). Previous studies have revealed that various miRNAs regulate the development of diabetes $(12,13)$. Downregulation of miR-15a in the retina of patients with diabetes was found to counteract inflammation and generation of vessels (14). A study on mice with diabetes showed that the expression of miR92a increased OS and inhibited the activity of ECs by binding to heme oxygenase-1 (15). miR-383 expression has been found to be suppressed before the onset of diabetes and to modulate death of pancreatic beta cells (16). miR-383 is also able to modulate cell death in various cancers $(17,18)$. Expression of miR-383 triggers the death of breast cancer cells by promoting their sensitivity to DNA injury (19). Nevertheless, investigation of the expression patterns of miR-383 in the endothelium of patients with diabetes is insufficient.

In this study, we examined the expression of miR-383 in ECs in response to high glucose and determined the function of miR-383 in high glucose-induced apoptosis and oxidative stress in ECs. Furthermore, the target genes mediating the function of miR-383 in ECs were identified. 


\section{Material and Methods}

\section{Animals}

C57BL/6 mice (glucose: $5 \pm 0.5 \mathrm{mmol} / \mathrm{L}, \mathrm{WT}, \mathrm{n}=4$, male, 8 weeks old, Shanghai SLAC Laboratory Animal Co., Ltd., China) and db/db (Obese Leptin resistant mice suffering from type 2 diabetes) mice (glucose: $25 \pm 2.1$ $\mathrm{mmol} / \mathrm{LL} \mathrm{n}=4$, male, 8 weeks, Shanghai SLAC Laboratory Animal Co., Ltd.) were housed at the regulated conditions of $22-23^{\circ} \mathrm{C}, 55 \pm 5 \%$ humidity, and a $12-\mathrm{h}$ light/dark cycle, with standard laboratory diet (Research Diet, Inc., USA) and water available ad libitum. All procedures related to animals complied with the Animal Experimentation Ethics Committee of Zibo Central Hospital.

\section{EC cultivation and high glucose supplementation}

Human umbilical vein endothelial cells (HUVECs) (Lonza, USA) were cultivated in EC proliferation media (CC3024; Lonza) containing antibiotics and 10\% FBS. Cells from passages 4 through 8 were used in further assays. As performed in previous studies, to prepare high glucose (HG) supplemented cell cultures, HUVECs were treated with D-glucose for $48 \mathrm{~h}$, followed by investigation of miR-383 expression, cell death, and generation of ROS (20).

\section{Qualitative polymerase chain reaction ( $q P C R$ ) of miR-} 383

Mice were anesthetized with an intraperitoneal injection of $350 \mathrm{mg}$ chloral hydrate per $\mathrm{kg}$ animal body weight and decapitated. The thoracic aorta was quickly dissected out of the mouse chest, and the surrounding fat/connective tissue was removed in Krebs-Henseleit $(\mathrm{KH})$ solution (mM: $118.0 \mathrm{NaCl}, 2.52 \mathrm{CaCl}_{2}, 1.16 \mathrm{MgSO}_{4}, 24.88$ $\mathrm{NaHCO}_{3}, 1.18 \mathrm{~K}_{2} \mathrm{PO}_{4}, 4.7 \mathrm{KCl}, 10.0$ glucose, 2.0 pyruvic acid, and 0.5 EDTA). Total RNA was isolated from HUVECs and aorta using TRIzol Reagent (Invitrogen, USA). RT-qPCR was carried out using Fast SYBR Green Master Mix (Applied Biosystems, USA) (21). The PCR cycling conditions were as follows: $5 \mathrm{~min}$ at $95^{\circ} \mathrm{C}, 36$ cycles: $10 \mathrm{~s}$ at $95^{\circ} \mathrm{C}, 10 \mathrm{~s}$ at $58^{\circ} \mathrm{C}$, and $20 \mathrm{~s}$ at $72^{\circ} \mathrm{C}$. Normalization of concentration of miR-383 to U6 concentration was carried out. The sequence of the miR-383 primer was forward: 5'-GTGCAGGGTCCGAGGT-3, reverse: 5'AGATCAGAAGGTGATTGTGGCT-3. The sequence of the U6 primer was forward: 5'-CTCGCTTCGGCAGCACA-3; reverse: 5'-AACGCTTCACGAATTTGCGT-3. The relative quantification of the target gene was conducted using the $2^{-\Delta \Delta \mathrm{Cq}}$ method. U6 was used as the internal control for miRNA.

\section{Transfection of HUVECs with miR-383 suppressor}

HUVECs were grown in 6-well plates at a density of $2 \times 10^{5}$ cells/well for $24 \mathrm{~h}$. The cells were then transfected with $80 \mathrm{nM}$ miR-383 suppressor (5-AGCCACAAUCACC UUCUGAUCU-3, NC (negative control): 5-CAGUACUUU UGUGUAGUACAA-3, GenePharma Co., Ltd, China), or
miR-383 mimic (5-AGAUCAGAAGGUGAUUGUGGCU-3, GenePharma Co., Ltd), or negative control (NC, 5-UUCU CCGAACGUGUCACGUTT-3) using Lipofectamine 2000 (Invitrogen) to knock down miR-383 expression. Cells proliferated in media without antibiotics for $24 \mathrm{~h}$ for use in additional assays. To assess knockdown of SIRT1, $40 \mathrm{nM}$ of SIRT1 siRNA (GenePharma Co., Ltd) or control siRNA was transfected into HUVECs using Lipofectamine RNAiMAX (ThermoFisher, USA). siRNA and $10 \mu \mathrm{L}$ of Lipofectamine RNAiMAX reagent (Invitrogen; Thermo Fisher Scientific, Inc.) was added to the culture medium and incubated for $6 \mathrm{~h}$ at $37^{\circ} \mathrm{C}$. Subsequently, the transfection mixture was removed and cells were further incubated with normal medium for a further $24 \mathrm{~h}$ at $37^{\circ} \mathrm{C}$. si-SIRT1 sense: 5'-CCGUCUCUGUGUCACAAAUTT-3', si-SIRT1 anti-sense: 5'-AUUUGUGACACAGAGACGGTT-3'; control siRNA sense: 5'-UUCUCCGAACGUGUCACGUTT-3', control siRNA anti-sense: 5'-ACGUGACACGUUCGGAGAATT-3'.

\section{Luciferase assay}

HUVECs were co-transfected with pGL3-SIRT1, a luciferase plasmid containing SIRT1 3'UTR, and miR-383 or its suppressor using the Neon Transfection System (ThermoFisher). After $48 \mathrm{~h}$, luciferase activities were measured with a Dual-Luciferase Reporter Assay System (Promega, USA). Firefly luciferase activity was normalized to Renilla luciferase activity.

\section{Assessment of cell death}

HUVECs were collected and centrifuged at $1000 \mathrm{~g}$ for $5 \mathrm{~min}$ at $4^{\circ} \mathrm{C}$ for supernatant elution. Binding buffer was used to resuspend the pellet. FITC annexin $V$ and propidium iodide (PI) were added prior to a 10-min incubation at room temperature. A FACScan flow cytometer (Becton, Dickinson and Company, USA) was used to examine fluorescent signals to assess cell death.

\section{Cell counting kit-8 (CCK-8) assay}

The viability of the cells was assessed using the CCK8 assay. In brief, at the predetermined time prior to the end of treatment, $100 \mu \mathrm{L}$ of CCK-8 solution was added to each well, and the cells were incubated at $37^{\circ} \mathrm{C}$ or $4 \mathrm{~h}$. The absorbance values at $450 \mathrm{~nm}$ were measured using a multi-well spectrophotometer (Bio-Rad, USA) at $450 \mathrm{~nm}$.

\section{Detection of catalase (CAT) and superoxide dismutase (SOD1) activity}

CAT and SOD1 activities were measured using a catalase assay kit and SOD assay kit (Cayman Chemical Co., USA) according to the manufacturer's instructions. The assay system consisted of $100 \mathrm{mM}$ PBS (pH 7.0, $100 \mu \mathrm{L})$, methanol $(30 \mu \mathrm{L})$ and sample $(20 \mu \mathrm{L}$ HUVEC from mouse aortas). The reaction was started by adding $35 \mu \mathrm{M} \mathrm{H} \mathrm{H}_{2} \mathrm{O}_{2}$ and the reaction mixture was incubated for $20 \mathrm{~min}$ at room temperature. After incubation, $10 \mathrm{M}$ potassium hydroxide and chromogen were added to the 
mixture. After further incubation for $10 \mathrm{~min}$, potassium periodate was added and incubated for $5 \mathrm{~min}$ at room temperature before reading the absorbance at $540 \mathrm{~nm}$ using a plate reader (Bio-Rad). CAT and SOD1 activities were calculated using the equation obtained from the linear regression of the standard curve.

\section{Detection of intracellular ROS}

HUVECs were incubated for $30 \mathrm{~min}$ with $25 \mu \mathrm{mol}$ fluorescent probe CM-H2DCFDA, then washed twice with PBS. To detect ROS inside cells, a multi-well fluorescent spectrophotometer was used at absorbance $485-530 \mathrm{~nm}$. The intensity of generation of ROS in control group was manually set at $100 \%$.

\section{Western blot analysis}

HUVECs were homogenized using lysis buffer (Beyotime, China) and proteins were separated using $8-15 \%$ SDS-PAGE. The proteins were transferred to PVDF membranes (Millipore, USA), blocked for $1 \mathrm{~h}$ in $5 \%$ milk, and incubated with anti- $\beta$-actin and anti-SIRT1 (Cell Signaling Technology, USA) primary antibodies (mouse anti-SIRT1, 2028, 1:1000, Cell Signaling; mouse anti- $\beta$-actin, 3700, $1: 5000$, Cell Signaling) overnight at $4^{\circ} \mathrm{C}$. Membranes were washed with PBS and then incubated with HRP-conjugated secondary antibodies (goat anti-mouse, 31430, $1: 8000$, ThermoFisher) for $1 \mathrm{~h}$ at $25^{\circ} \mathrm{C}$. Enhanced chemiluminescence reagent (ThermoFisher Pierce) was applied for protein band detection. Beta-actin was used as a loading control for normalization in western blotting.

\section{Statistical analysis}

Statistical analysis was performed by SPSS Statistics version 17.0 (USA) using ANOVA and Tukey's post hoc test. The results are reported as means $\pm S E$, with $\mathrm{P}<0.05$ considered significant.

\section{Results}

miR-383 expression was promoted in diabetic murine aortas and in ECs exposed to HG

To examine the expression of miR-383 in diabetic mouse endothelial cell, RT-qPCR of miR-383 was carried out in $\mathrm{db} / \mathrm{db}$ mice and HUVECs that received $\mathrm{HG}$ in vitro. As presented in Figure 1, miR-383 expression was increased in the aortas of mice with diabetes compared to wild type (WT) mice. Additionally, miR-383 expression was higher in HUVECs treated with HG than in control cells.
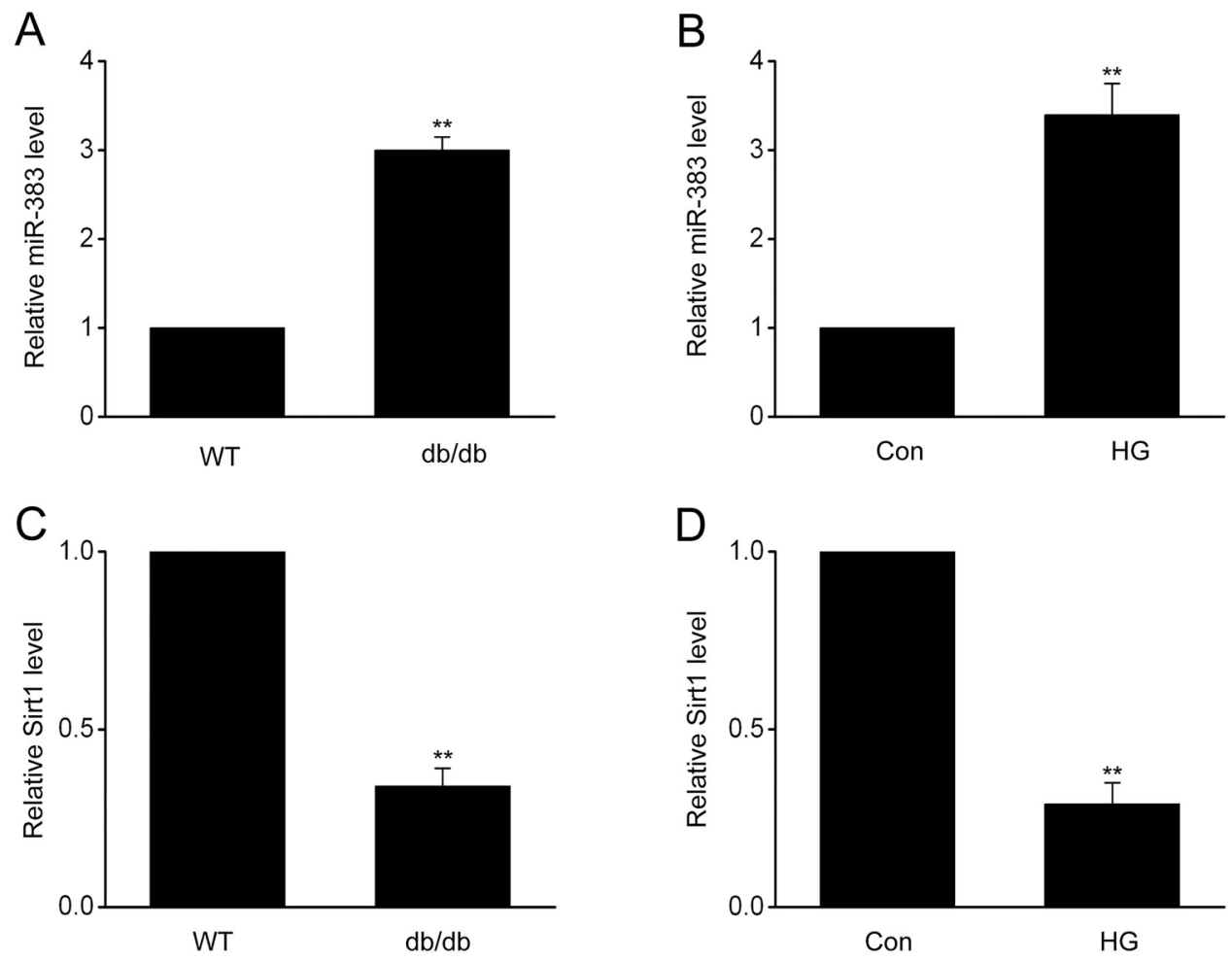

Figure 1. miR-383 expression was promoted in diabetic mice. Increased expression of miR-383 in (A) aortas of $\mathrm{db} / \mathrm{db}$ mice and (B) human umbilical vein endothelial cells (HUVECs) supplemented with high glucose (HG) was evaluated by qRT-PCR. Knocked-down expression of SIRT1 in (C) aortas of db/db mice and (D) HUVECs supplemented with HG was evaluated by qRT-PCR. Data are reported as means \pm SE of 3 independent experiments. ${ }^{* *} \mathrm{P}<0.01$ (ANOVA and Tukey's post hoc test). WT: wild type; Con: control. 
A

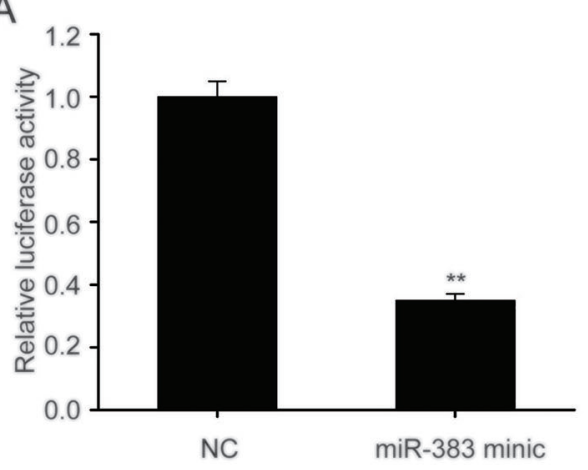

C

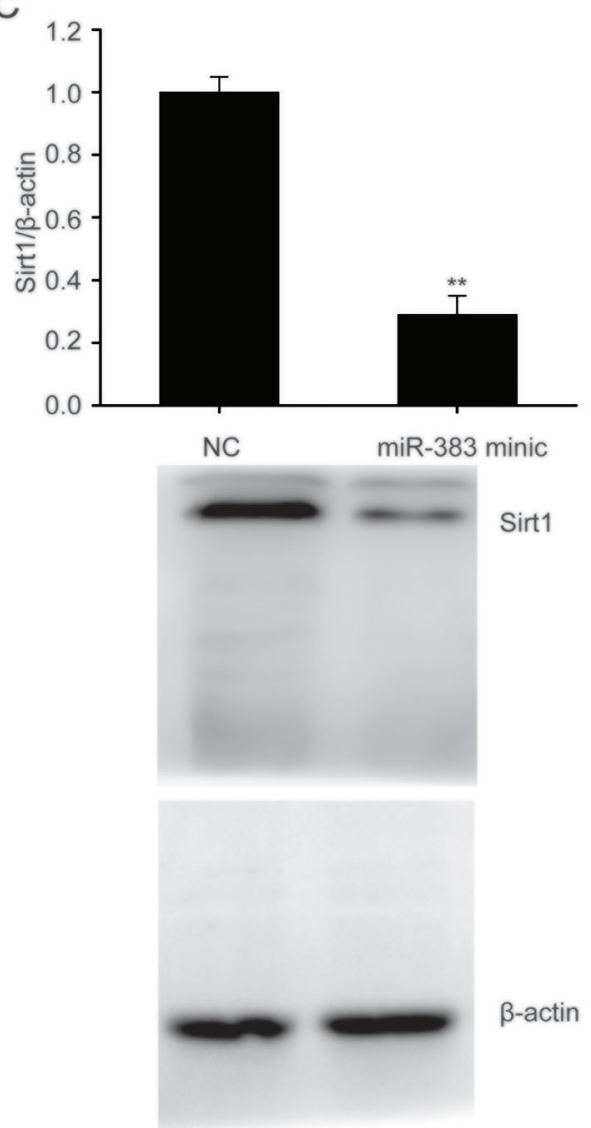

B

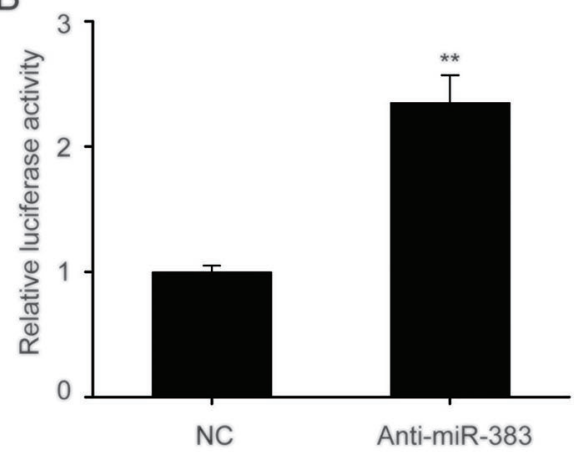

D

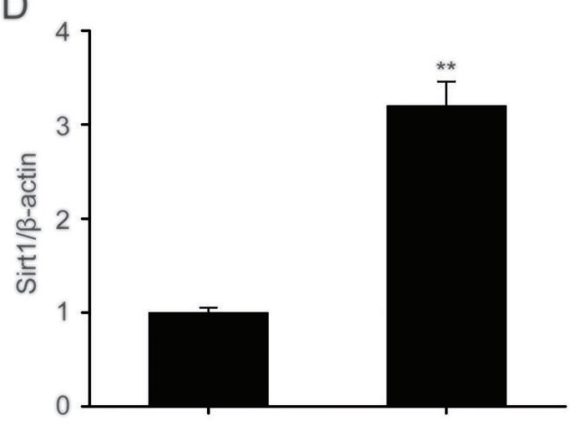

NC Anti-miR-383

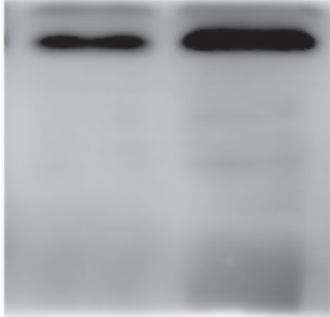

Sirt1

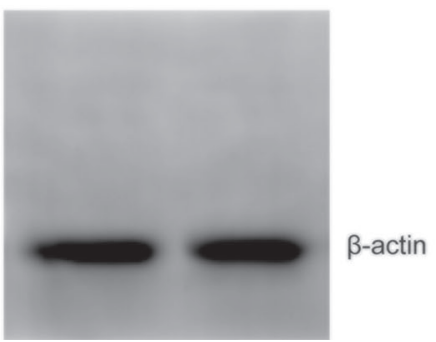

Figure 2. A and B, Luciferase function assay was used to examine the correlation between miR-383 and SIRT1. C and D, Representative immunoblots and quantitative evaluation of SIRT1 in human umbilical vein endothelial cells transfected with miR-383 mimic or miR-383 suppressor for $24 \mathrm{~h}$. Data are reported as means \pm SE of 3 independent experiments. ${ }^{* *} \mathrm{P}<0.01$ (ANOVA and Tukey's post hoc test). NC: negative control.

Furthermore, expression of SIRT1 was suppressed in $\mathrm{db} / \mathrm{db}$ mice and HUVECs treated with HG compared to controls. These findings indicated that increased expression of miR-383 may be related to the malfunction of ECs under diabetic conditions.

\section{miR-383 targeted SIRT1 in ECs}

The miRanda database (www.microrna.org) was used to filter and identify the putative target genes of miR-383. Bioinformatics analysis predicted that SIRT1 may serve as a target of miR-383. We found that increased expression 
of miR-383 suppressed the expression of SIRT1 in HUVECs and suppression of miR-383 promoted its expression (Figure 2A and $B$ ). Expression of SIRT1 was likely regulated by miR-383 binding directly to the $3^{\prime} U T R$ and prohibiting translation. Suppression of miR-383 enhanced the expression of the SIRT1 protein in HUVECs (Figure 2C and D).

\section{Suppression of miR-383 decreased OS in ECs}

It has been shown that increased OS contributes to the dysfunction of ECs under diabetic conditions (3). To investigate the influence of miR-383 on OS, we carried out loss of function assays by transfecting HUVECs with miR-383 suppressor or NC. Staining with CM-H2DCFDA fluorescent probe showed that ROS concentration increased in the HUVECs transfected with NC (Figure $3 A$ and $B$ ). miR-383 suppression significantly repressed the generation of ROS in HUVECs. Furthermore, miR-383 suppression elevated CAT and SOD1 activity, as effective endogenous antioxidant, compared to the NC (Figure $3 \mathrm{C}$ and $\mathrm{D}$ ). In addition, miR-383 suppression increased the activity of CAT and SOD1 in the aortas of $\mathrm{db} / \mathrm{db}$ mice (Figure 3E and F). These findings proved that suppression of miR-383 represses excessive ROS generation in ECs under diabetic conditions.

\section{Suppression of miR-383 reduced cell death of ECs}

OS stimulation triggered by HG is an essential mechanism in ECs death (22). Thus, we examined the influence of miR-383 suppression on the death of HUVECs.
A

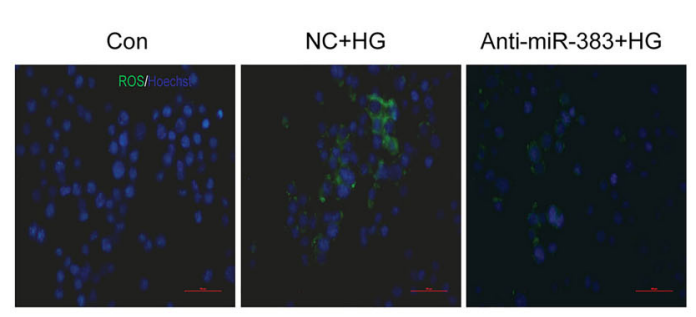

C

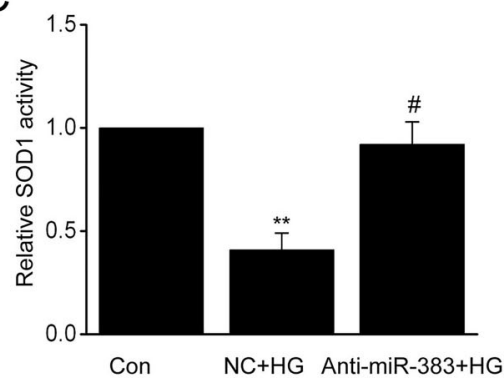

E

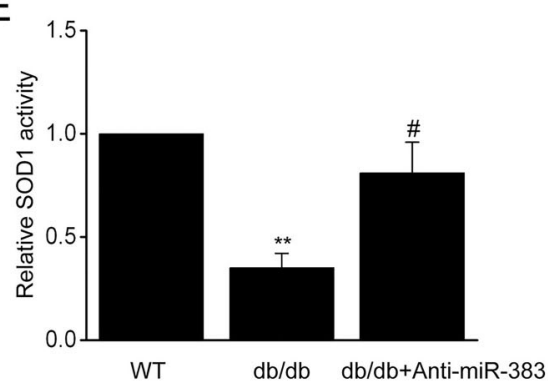

B

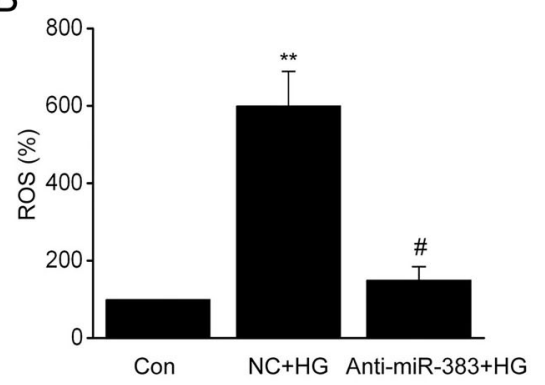

D

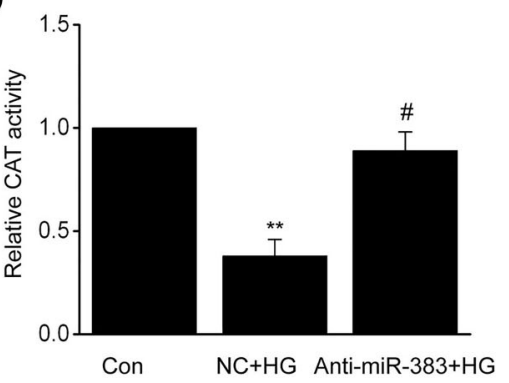

$\mathrm{F}$

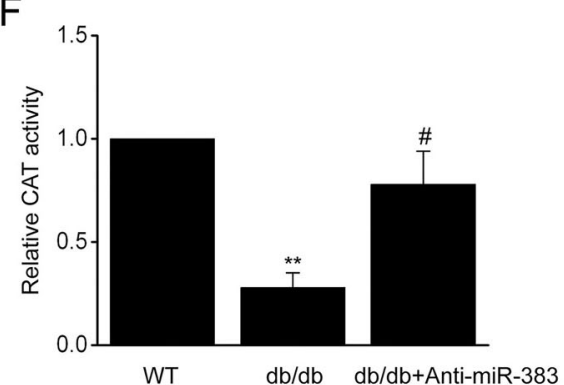

Figure 3. Human umbilical vein endothelial cells were transfected with miR-383 suppressor or negative control (NC) for $24 \mathrm{~h}$ before $48 \mathrm{~h}$ activation by treatment with high glucose (HG). A, Reactive oxygen species (ROS) inside the cells was assessed by the oxidation of H2DCFDA to DCF (magnification bars: $40 \mu \mathrm{m}$ ). B, Quantification of ROS. C, Activity of superoxide dismutase (SOD1) and D, catalase (CAT). E, Activity of SOD1 and F, CAT in aortas of $\mathrm{db} / \mathrm{db}$ mice. Data are reported as mean $\pm \mathrm{SE}$ of 3 independent experiments. ${ }^{* *} \mathrm{P}<0.01$ vs control or WT; ${ }^{\#} \mathrm{P}<0.05$ vs NC + HG group or db/db (ANOVA and Tukey's post hoc test). WT: wild type; Con: control. 


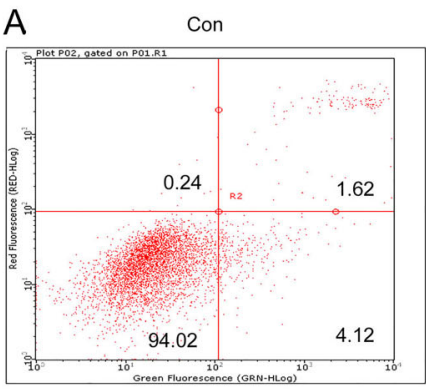

B

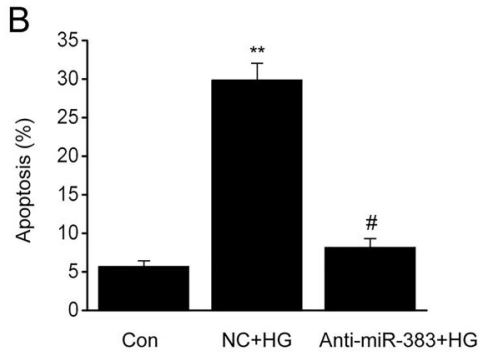

$\mathrm{NC}+\mathrm{HG}$

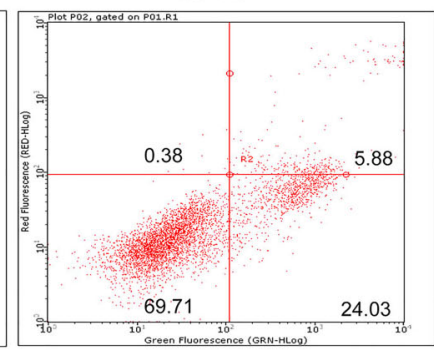

Anti-miR-383+HG

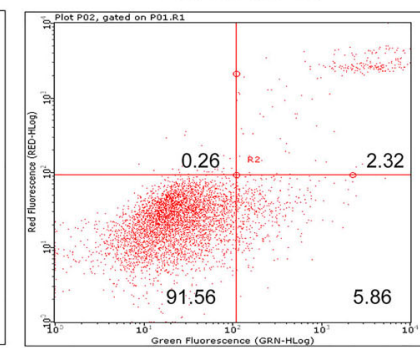

C

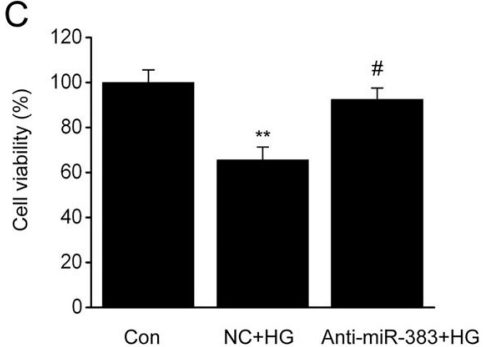

Figure 4. Human umbilical vein endothelial cells were transfected with miR-383 suppressor or negative control (NC) for $24 \mathrm{~h}$ prior to $48 \mathrm{~h}$ activation by treatment with high glucose (HG). A, Flow cytometry analysis of cell death. B, Quantification of cell death in each group. C, Cell viability was assessed by CCK-8 assay. Data are reported as means $\pm \mathrm{SE}$ of 3 independent experiments. ${ }^{\star *} \mathrm{P}<0.01$ vs control; ${ }^{\#} \mathrm{P}<0.05$ vs NC + HG group (ANOVA and Tukey's post hoc test). Con: control.

Supplementation of the cells with $\mathrm{HG}$ increased the quantity of dead cells, which was counteracted by suppression of miR-383 (Figure 4A-C). Thus, targeting miR-383 could promote the viability of EC cells exposed to $\mathrm{HG}$.

SIRT1 knockdown counteracted the influence of miR383 suppression on ROS generation and ECs death

To examine if SIRT1 regulated the influence of miR383 suppression on the attenuation of OS and promotion of EC viability, siRNA was used to knock down expression of SIRT1 in HUVECs (Figure 5A and B). The influence of miR-383 suppression on the ROS generation by HUVECs following exposure to $H G$ was counteracted by knockdown of SIRT1. Knockdown of SIRT1 repressed the reinforcement of CAT and SOD1 that is usually triggered by suppression of miR-383. Furthermore, the inhibitory influence of miR-383 suppression on HG-triggered death of HUVECs was counteracted by knockdown of SIRT1 (Figure 5C-F). These results showed that suppression of ROS generation and death of ECs by miR-383 can be modulated by knockdown of SIRT1.

\section{Discussion}

miRNAs are essential contributors to the modulation of CVD by targeting various genes $(23,24)$. miR-383 is involved in various cellular processes related to the development of various diseases, such as generation of malignancies and cerebral ischemia (25-27). Our study found an increased expression of miR-383 in murine aortic ECs from $\mathrm{db} / \mathrm{db}$ mice that were treated with $\mathrm{HG}$. It was revealed that increased expression of miR-383 in diabetic murine ECs reinforced OS, and suppression of miR-383 impaired cell death and normalized ROS generation in ECs under diabetic conditions.

miR-383 targeted SIRT1, whose upregulation promoted endothelial activity by suppressing miR-383 expression in $\mathrm{db} / \mathrm{db}$ mice. These findings emphasized the promising influence of miR-383 on regulation of malfunctioning vessels in patients suffering from diabetes. Targeting of miR-383 may be a way to treat vessel pathologies in diabetes.

Excessive ROS generation contributes to EC malfunction in diabetes (28). Previous studies have shown that ROS generation partly contributes to HG-triggered cell death of ECs $(29,30)$. OS normalization, resulting from use of antioxidants, reduces damage to vessels in patients with diabetes $(31,32)$. We found that suppression of miR-383 reduced ROS generation, elevated the levels SOD1/CAT, and repressed ECs death that were supplemented with HG. This indicated that endothelial activity was promoted through miR-383 suppression in ECs by prohibition of ROS under diabetic conditions.

Our study revealed the target gene that could participate in preserving the influence of miR-383 suppression on ECs in patients with diabetes. We screened for enzyme expression related to OS in HUVECs transfected with miR-383 suppressor and found promotion of SIRT1 translation. We confirmed that suppression of 
A
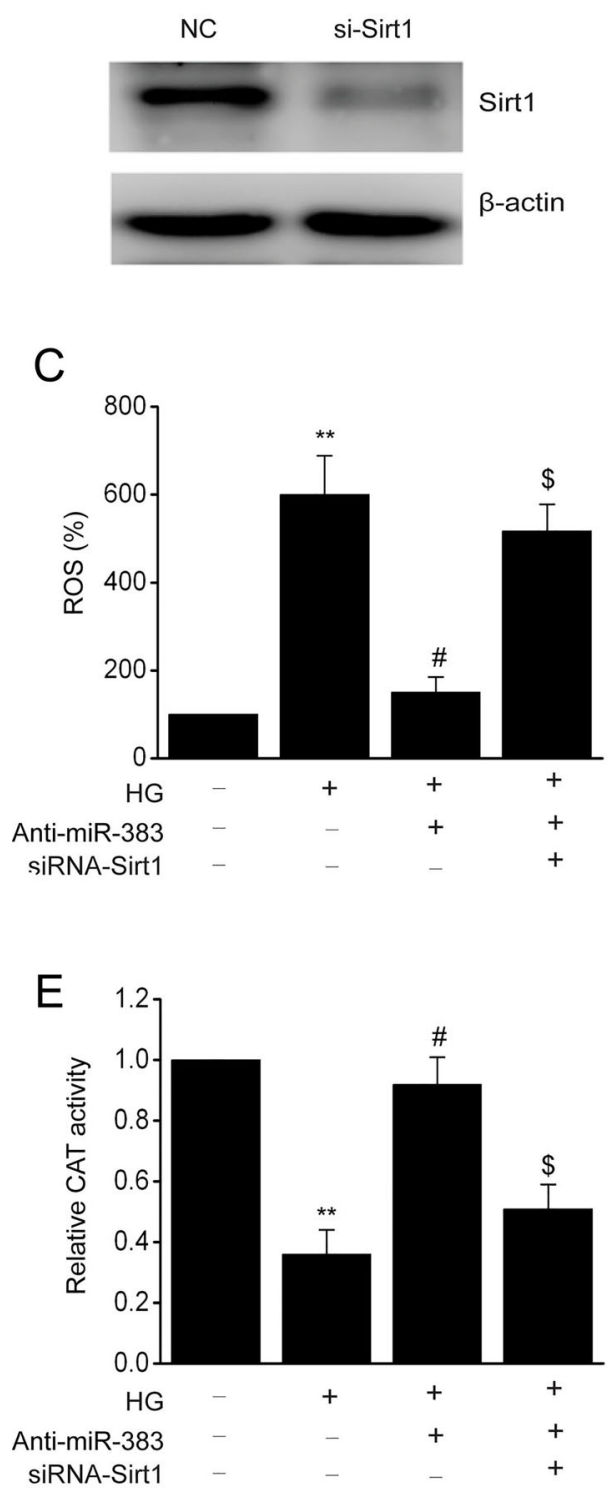

B

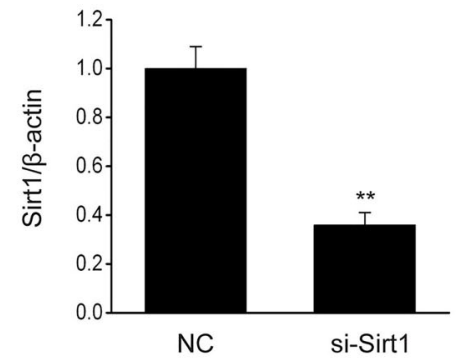

D
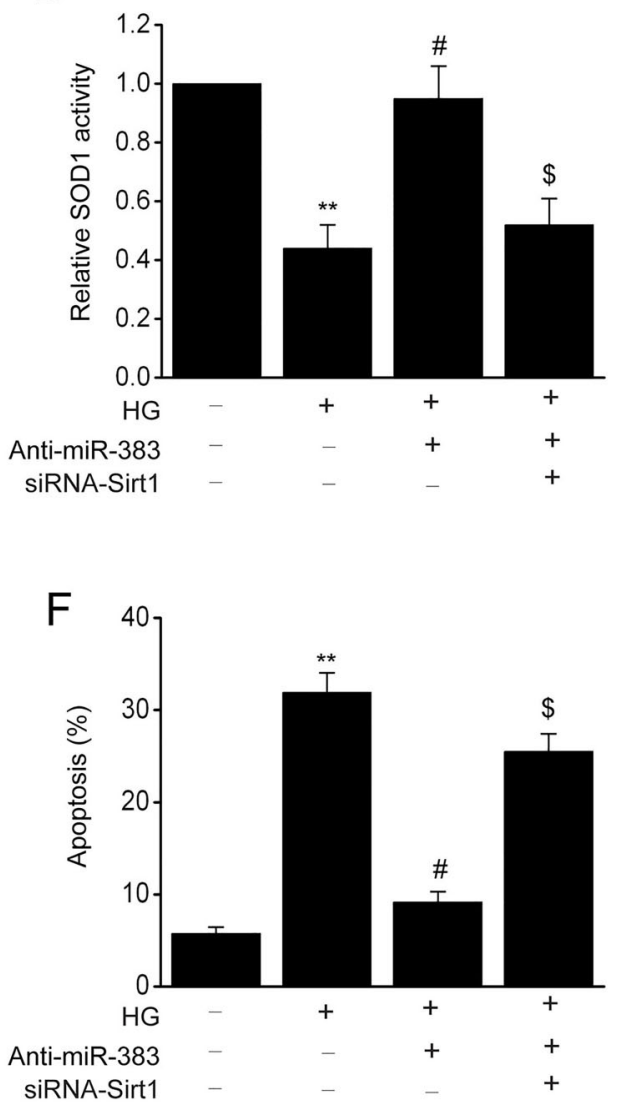

Figure 5. Human umbilical vein endothelial cells (HUVECs) were transfected with SIRT1 siRNA and miR-383 suppressor for $24 \mathrm{~h}$ prior to $48 \mathrm{~h}$ activation by treatment with high glucose (HG). A and B, Representative immunoblot and quantitative evaluation of SIRT1 in HUVECs. C, Reactive oxygen species (ROS) inside the cells was assessed by oxidation of H2DCFDA to DCF. D, Activity of superoxide dismutase (SOD1) and E, catalase (CAT) in groups. F, Flow cytometry analysis of cell death. Data are reported as means \pm SE of 3 independent experiments. ${ }^{* *} \mathrm{P}<0.01$ vs control; ${ }^{\#} \mathrm{P}<0.05$ vs negative control $(\mathrm{NC})+\mathrm{HG}$ group; ${ }^{\$} \mathrm{P}<0.05$ vs anti-miR-383 group (ANOVA and Tukey's post hoc test).

miR-383 directly promoted expression of SIRT1 in ECs. Although previous research has explored the etiology of SIRT1 modulation in ECs, it has not been well studied in diabetes $(33,34)$. In the present study, we found that SIRT1 was downregulated in HUVECs treated with HG. Downregulation of SIRT1 was also seen in ECs from the aortas of $d b / d b$ mice. Our research has shown that SIRT1 suppressed miR-383 expression in diabetic murine ECs to preserve their influence on vessels.

In summary, our study provided evidence that targeting miR-383/SIRT1 axis can be an alternative therapeutic strategy for ameliorating diabetic vascular degeneration. 


\section{References}

1. Cheng YC, Sheen JM, Hu WL, Hung YC. Polyphenols and oxidative stress in atherosclerosis-related ischemic heart disease and stroke. Oxid Med Cell Longev 2017; 2017: 8526438, doi: 10.1155/2017/8526438.

2. Cui K, Tang Z, Li CC, Wang T, Rao K, Wang SG, et al. Lipoxin A4 improves erectile dysfunction in rats with type I diabetes by inhibiting oxidative stress and corporal fibrosis. Asian J Androl 2018; 20: 166-172, doi: 10.4103/aja.aja_ 49 17.

3. Robson R, Kundur AR, Singh I. Oxidative stress biomarkers in type 2 diabetes mellitus for assessment of cardiovascular disease risk. Diabetes Metab Syndr 2018; 12: 455-462, doi: 10.1016/j.dsx.2017.12.029.

4. Wang S, Wang J, Zhao A, Li J. SIRT1 activation inhibits hyperglycemia-induced apoptosis by reducing oxidative stress and mitochondrial dysfunction in human endothelial cells. Mol Med Rep 2017; 16: 3331-3338, doi: 10.3892/mmr. 2017.7027.

5. Watt NT, Gage MC, Patel PA, Viswambharan H, Sukumar P, Galloway S, et al. Endothelial SHIP2 suppresses Nox2 NADPH oxidase-dependent vascular oxidative stress, endothelial dysfunction, and systemic insulin resistance. Diabetes 2017; 66: 2808-2821, doi: 10.2337/db17-0062.

6. Cao R, Wang G, Qian K, Chen L, Qian G, Xie C, et al. Silencing of HJURP induces dysregulation of cell cycle and ROS metabolism in bladder cancer cells via PPARgammaSIRT1 feedback loop. J Cancer 2017; 8: 2282-2295, doi: 10.7150/jca.19967.

7. Song SB, Jang SY, Kang HT, Wei B, Jeoun UW, Yoon GS, et al. Modulation of mitochondrial membrane potential and ROS generation by nicotinamide in a manner independent of SIRT1 and mitophagy. Mol Cells 2017; 40: 503-514, doi: 10.14348/molcells.2017.0075.

8. Sin TK, Yung BY, Siu PM. Modulation of SIRT1-Foxo1 signaling axis by resveratrol: implications in skeletal muscle aging and insulin resistance. Cell Physiol Biochem 2015; 35: 541-552, doi: 10.1159/000369718.

9. Xu X, Ji S, Li W, Yi B, Li H, Zhang H, et al. LncRNA H19 promotes the differentiation of bovine skeletal muscle satellite cells by suppressing Sirt1/FoxO1. Cell Mol Biol Lett 2017; 22: 10, doi: 10.1186/s11658-017-0040-6.

10. Perez-Sanchez C, Arias-de la Rosa I, Aguirre MA, LuqueTevar M, Ruiz-Limon P, Barbarroja N, et al. Circulating microRNAs as biomarkers of disease and typification of the atherothrombotic status in antiphospholipid syndrome. Haematologica 2018; 103: 908-918, doi: 10.3324/haematol. 2017.184416.

11. Mens MMJ, Ghanbari M. Cell cycle regulation of stem cells by MicroRNAs. Stem Cell Rev 2018; 14: 309-322, doi: 10.1007/s12015-018-9808-y.

12. Goncalves FZ, Lizarte Neto FS, Novais PC, Gattas D, Lourenco LG, de Carvalho CAM, et al. Expression profile of endothelin receptors (ETA and ETB) and microRNAs155 and -199 in the corpus cavernosum of rats submitted to chronic alcoholism and diabetes mellitus. Braz J Med Biol Res 2018; 51: e6329, doi: 10.1590/1414-431x2017 6329.

13. Houshmand-Oeregaard A, Schrolkamp M, Kelstrup L, Hansen NS, Hjort L, Thuesen ACB, et al. Increased expression of microRNA-15a and microRNA-15b in skeletal muscle from adult offspring of women with diabetes in pregnancy. Hum Mol Genet 2018; 27: 1763-1771, doi: 10.1093/hmg/ddy085.

14. Garcia-Diaz DF, Camacho-Guillen P, Codner E, PerezBravo F. miR15a and miR16 in Chilean type 1 diabetes patients: possible association with apoptosis, inflammatory, or autoimmunity markers. J Endocrinol Invest 2018; 41: 1083-1088, doi: 10.1007/s40618-018-0837-9.

15. Gou L, Zhao L, Song W, Wang L, Liu J, Zhang H, et al. Inhibition of miR-92a suppresses oxidative stress and improves endothelial function by upregulating heme oxygenase-1 in db/db mice. Antioxid Redox Signal 2018; 28: 358-370, doi: 10.1089/ars.2017.7005.

16. Xia SF, Duan XM, Cheng XR, Chen LM, Kang YJ, Wang P, et al. Role of miR-383 and miR-146b in different propensities to obesity in male mice. J Endocrinol 2017; 234: 201-216, doi: 10.1530/JOE-17-0044

17. Azarbarzin S, Feizi MAH, Safaralizadeh R, Kazemzadeh M, Fateh A. The value of MiR-383, an intronic MiRNA, as a diagnostic and prognostic biomarker in intestinal-type gastric cancer. Biochem Genet 2017; 55: 244-252, doi: 10.1007/s10528-017-9793-x.

18. Chen L, Guan H, Gu C, Cao Y, Shao J, Wang F. miR-383 inhibits hepatocellular carcinoma cell proliferation via targeting APRIL. Tumour Biol 2016; 37: 2497-2507, doi: 10.1007/ s13277-015-4071-1.

19. Lee $\mathrm{CH}$, Kuo WH, Lin CC, Oyang YJ, Huang HC, Juan HF. MicroRNA-regulated protein-protein interaction networks and their functions in breast cancer. Int $J$ Mol Sci 2013; 14: 11560-11606, doi: 10.3390/ijms140611560.

20. Chang KC, Snow A, LaBarbera DV, Petrash JM. Aldose reductase inhibition alleviates hyperglycemic effects on human retinal pigment epithelial cells. Chem Biol Interact 2015; 234: 254-260, doi: 10.1016/j.cbi.2014.10.007.

21. Zhao L, Gu H, Chang J, Wu J, Wang D, Chen S, et al. MicroRNA-383 regulates the apoptosis of tumor cells through targeting Gadd45g. PLoS One 2014; 9: e110472, doi: 10.1371/journal.pone.0110472.

22. Norouzirad R, Gonzalez-Muniesa P, Ghasemi A. Hypoxia in obesity and diabetes: potential therapeutic effects of hyperoxia and nitrate. Oxid Med Cell Longev 2017; 2017: 5350267, doi: 10.1155/2017/5350267.

23. Gong YY, Luo JY, Wang L, Huang Y. MicroRNAs Regulating reactive oxygen species in cardiovascular diseases. Antioxid Redox Signal 2018; 29: 1092-1107, doi: 10.1089/ars. 2017.7328

24. Romakina VV, Zhirov IV, Nasonova SN, Zaseeva AV, Kochetov AG, Liang OV, et al. MicroRNAs as biomarkers of cardiovascular diseases [in Russian]. Kardiologiia 2018; 66-71, doi: 10.18087/cardio.2018.1.10083.

25. Fang Z, He L, Jia H, Huang $Q$, Chen D, Zhang Z. The miR383-LDHA axis regulates cell proliferation, invasion and glycolysis in hepatocellular cancer. Iran J Basic Med Sci 2017; 20: 187-192, doi: 10.22038/ijbms.2017.8246.

26. Han RL, Wang FP, Zhang PA, Zhou XY, Li Y. miR-383 inhibits ovarian cancer cell proliferation, invasion and aerobic glycolysis by targeting LDHA. Neoplasma 2017; 64: 244-252, doi: 10.4149/neo_2017_211. 
27. Li Y, Ning Q, Shi J, Chen Y, Jiang M, Gao L, et al. A novel epigenetic AML1-ETO/THAP10/miR-383 mini-circuitry contributes to $\mathrm{t}(8 ; 21)$ leukaemogenesis. EMBO Mol Med 2017; 9: 933-949, doi: 10.15252/emmm.201607 180.

28. Magenta A, Greco S, Capogrossi MC, Gaetano C, Martelli F. Nitric oxide, oxidative stress, and p66Shc interplay in diabetic endothelial dysfunction. Biomed Res Int 2014; 2014: 193095, doi: 10.1155/2014/193095.

29. Rezende F, Moll F, Walter $M$, Helfinger $V$, Hahner $F$, Janetzko $P$, et al. The NADPH organizers NoxO1 and p47phox are both mediators of diabetes-induced vascular dysfunction in mice. Redox Biol 2018; 15: 12-21, doi: 10.1016/j.redox.2017.11.014.

30. Wang X, Qin J, Zhang X, Peng Z, Ye K, Wu X, et al. Functional blocking of Ninjurin1 as a strategy for protecting endothelial cells in diabetes mellitus. Clin Sci (Lond) 2018; 132: 213-229.
31. Ambasta RK, Kohli H, Kumar P. Multiple therapeutic effect of endothelial progenitor cell regulated by drugs in diabetes and diabetes related disorder. J Trans/ Med 2017; 15: 185, doi: 10.1186/s12967-017-1280-y.

32. Du JY, Yuan F, Zhao LY, Zhu J, Huang YY, Zhang GS, et al. Suppression of Kv1.5 protects against endothelial apoptosis induced by palmitate and in type 2 diabetes mice. Life Sci 2017; 168: 28-37, doi: 10.1016/j.lfs.2015.12.054.

33. Hu Q, Wang G, Peng J, Qian G, Jiang W, Xie C, et al. Knockdown of SIRT1 suppresses bladder cancer cell proliferation and migration and induces cell cycle arrest and antioxidant response through $\mathrm{FOXO} a \mathrm{a}-m e d i a t e d$ pathways. Biomed Res Int 2017; 2017: 3781904, doi: 10.1155/ 2017/3781904.

34. Singh CK, Chhabra G, Ndiaye MA, Garcia-Peterson LM, Mack NJ, Ahmad N. The role of sirtuins in antioxidant and redox signaling. Antioxid Redox Signal 2018; 28: 643-661, doi: 10.1089/ars.2017.7290. 